\title{
Uso de inhibidores selectivos de la recaptación de serotonina y defectos específicos de nacimiento
}

\author{
Use of selective serotonins reuptake inhibitors and birth defects
}

\section{Objetivo}

Evaluar la asociación entre el uso perinatal de inhibidores selectivos de la recaptación de la serotonina (ISRS) y los defectos de nacimiento.

\section{Diseño, lugar y pacientes}

Análisis bayesiano para combinar los resultados de los análisis independientes publicados, con los datos de una base originada en un estudio multicéntrico de casos y control de defectos de nacimiento en la población que había recolectado información de diez centros de Estados Unidos entre 1997 y 2009.

Participaron 17.952 madres de bebés con defectos de nacimiento y 9.857 madres de bebés sin ellos, identificadas a través de certificados o de registros hospitalarios de nacimientos.

\section{Evaluación de factores pronósticos}

Se evaluó la exposición de la población a citalopram, escitalopram, fluoxetina, paroxetina y sertralina, antes y durante el tercer mes de embarazo. Mediante el uso de la razón de odds (odds ratio) fueron realizadas estimaciones posteriores, ajustándolas de acuerdo a variables maternas como el tabaquismo y la obesidad antes del embarazo, la raza y el nivel educativo.

\section{Medición de resultados principales}

Los 14 defectos de nacimiento potencialmente asociados con el uso de ISRS según reportes bibliográficos.
Resultados principales

La sertralina fue el ISRS más frecuentemente usado, sin que pudiera confirmarse ninguna de las cinco potenciales asociaciones con defectos de nacimiento reportadas previamente. Se observaron altas probabilidades de asociación entre cinco defectos de nacimiento y el uso de paroxetina y fluoxetina. Ver tabla 1.

Tabla 1: principales asociaciones detectadas entre inhibidores selectivos de la recaptación de serotonina y defectos del nacimiento.

\begin{tabular}{c|l|c} 
Droga & \multicolumn{1}{|c|}{ Defecto } & OR (IC95\%) \\
\hline Paroxetina & Ónfalocele & $3,5(1,3 \mathrm{a} 8,0)$ \\
& Anencefalia & $3,2(1,06 \mathrm{a} 6,02)$ \\
& Gastrosquisis & $2,5(1,2 \mathrm{a} 4,8)$ \\
& Obstrucción del tracto de salida del ventrículo derecho & $2,4(1,4 \mathrm{a} 3,9)$ \\
& Defectos del tabique auricular & $1,8(1,1 \mathrm{a} 3,0)$ \\
\hline Fluoxetina & Obstrucción del tracto de salida del ventrí́culo derecho & $2,0(1,4 \mathrm{a} 3,1)$ \\
& Craniosinostosis & $1,9(1,1 \mathrm{a} 3,0)$ \\
\hline
\end{tabular}

\section{Conclusiones}

Estos datos proporcionan evidencia tranquilizadora para el uso de algunos ISRS, pero sugieren que algunos defectos de nacimiento aumentan su probabilidad de ocurrencia en las mujeres tratadas con paroxetina o fluoxetina durante fases tempranas del embarazo.

Fuente de financiamiento: Centros de Prevención y Control de las Enfermedades de EE.UU.

\section{Comentario}

En 1996 el Congreso de los estados Unidos ordenó a los Centros para el Control y Prevención de Enfermedades (CDC) establecer los Centros de Investigación y Prevención de los Defectos de Nacimiento (CBDRP). Los mismos están ubicados en Arkansas, California, Georgia, lowa, Massachusetts, Nueva Jersey, Nueva York, Carolina del Norte, Texas y Utah. Estos estados tienen programas con experiencia reconocida a nivel nacional sobre la vigilancia y la investigación de defectos de nacimiento y sus centros han entrevistado a más de 35.000 mujeres y además han recolectado material genético de las mejillas de las familias para ampliar las investigaciones'.

En términos generales, los resultados de esta investigación guardan relación con hallazgos previos en países nórdicos
(Dinamarca, Finlandia, Islandia, Noruega y Suecia) ${ }^{2}$. Sin embargo, estos autores no habían comunicado incremento de riesgos asociados al uso de paroxetina y fluoxetina.

\section{Conclusión de la comentadora}

La decisión de medicar con un ISRS nunca está libre de riesgos durante el embarazo. Para ello debe evaluarse cada situación clínica, teniendo en cuenta las posibles consecuencias de su uso como las derivadas de la decisión de no medicar adecuadamente. La información que brindan las investigaciones nos permiten tomar decisiones en base a la evidencia, tratando en lo posible de elegir entre los ISRS cuyos efectos teratogénicos no hayan sido documentados.

Silvina Foks [ Servicio de Psiquiatría del Hospital Italiano de Buenos Aires. ivyfo@yahoo.es]

Foks S. Uso de inhibidores selectivos de la recaptación de serotonina y defectos específicos de nacimiento. Evid Act Pract Ambul. 2016;19(2):55. AbrJun. Comentado de: Reefhuis $\mathrm{J}$ y col. Specific SSRIs and birth defects: bayesian analysis to interpret new data in the context of previous reports. BMJ 2015;350:h3190 doi: 10.1136/bmj.h3190

Referencias

1. Sitio oficial del National Birth Prevention Study. Disponible en URL: http://www.nbdps.org (último acceso: 23/09/15)

2. Stephansson O y col. Al. Selective Serotonin Reuptake Inhibitors During Pregnancy and Risk of Stillbirth and Infant Mortality. JAMA. 2013 Jan 2;309(1):48-54. doi: 10.1001/jama.2012.153812. 